\title{
On Ciric's fixed point theorem
}

by

Barada K. Ray (Durgapur)

Abstract. Some results related to a fixed point theorem of L. B. Ciric have been presented in this paper.

0. Let $(X, d)$ be a complete metric space and let $T$ be a mapping of $X$ into itself such that

$$
d(T x, T y) \leqslant \alpha d(x, y)
$$

where $0 \leqslant \alpha<1$ and $x, y \in X$. Then by Banach's [2] fixed point theorem $T$ has a unique fixed point. According to Kannan's [3] fixed point theorem the following condition also implies that $T$ has a unique fixed point:

$$
d(T x, T y) \leqslant \alpha[d(x, T x)+d(y, T y)]
$$

where $0 \leqslant \alpha<\frac{1}{2}$ and $x, y \in X$. Recently Chatterjee [4] has proved that if $T_{1}$ and $T_{2}$ be two selfmappings of a complete metric space $X$ such that

$$
d\left(T_{1} x, T_{2} y\right) \leqslant \alpha\left[d\left(x, T_{2} y\right)+d\left(y, T_{1} x\right)\right]
$$

for all $x, y$ in $X$ and for some $\alpha$ with $0<\alpha<\frac{1}{2}$, then $T_{1}$ and $T_{2}$ have a unique common fixed point. If we take $T_{1}=T_{2}=T$ in the result of Chatterjee, then as a Corollary we get the following:

If $T$ be a selfmapping of a complete metric space $X$ such that

$$
d(T x, T y) \leqslant \alpha[d(x, T y)+d(y, T x)]
$$

where $x, y \in X, 0<\alpha<\frac{1}{2}$, then $T$ has a unique fixed point.

These results we unified in [1] where Ciric proved:

THEOREM 1. If $T$ be a selfmapping of a complete metric space $X$ such that

(5) $\quad d(T x, T y) \leqslant \alpha d(x, y)+\beta[d(x, T x)+d(y, T y)]+\gamma[d(x, T y)+\dot{d}(y, T x)]$

for all $x, y$ in $X$ and for some $\alpha, \beta, \gamma \in R_{+}$with $\alpha+2 \beta+2 \gamma<1$, then $T$ has a unique fixed point.

We [11] have recently established the following:

4 - Fundamenta Mathematicae XCIV 
Let $T_{1}$ and $T_{2}$ be two selfmappings of a complete metric space $(X, d)$ such that (6) $\quad d\left(T_{1}^{p} x, T_{2}^{q} y\right) \leqslant \alpha d(x, y)+\beta d\left(x, T_{1}^{p} x\right)+\gamma d\left(y, T_{2}^{q} y\right)+\delta\left[d\left(x, T_{2}^{q} y\right)+d\left(y, T_{1}^{p} x\right)\right]$ for all $x, y \in X$ where $\alpha, \beta, \gamma, \delta \in R_{+}$with $\alpha+\beta+\gamma+2 \delta<1$ and $p, q$ are positive integers, then $T_{1}$ and $T_{2}$ have a unique common fixed point.

If we take $T_{1}=T_{2}=T$ and $p=q=1$ in (6) then as a Corollaıy we get the following theorem:

THeORem 2. Let $T$ be a selfmapping of a metric space $X$ (complete) such that (7) $\quad d(T x, T y) \leqslant \alpha d(x, y)+\beta d(x, T x)+\gamma d(y, T y)+\delta[d(x, T y)+d(y, T x)]$

for all $x, y \in X$ and for some $\alpha, \beta, \gamma, \delta \in R_{+}$with $\alpha+\beta+\gamma+2 \delta<1$, then Thas a unique fixed point. in (7).

We note that the theorem of Ciric follows from Theorem 2 by taking $\beta=\gamma$

The aim of this paper is to generalize Theorem 1 in different directions. A few theorems on sequence of mappings have also been presented in this paper. Throughout this paper $X$ will denote a complete metric space and $d$ the metric on $X$.

1. Rakotch [5] proved the following result.

THEOREM 3. Let $T$ be a selfmapping of $X$ such that

$$
d(T x, T y) \leqslant \alpha(d(x, y)) d(x, y) \quad \text { for each } x \neq y \in X
$$

where $\alpha:(0, \infty) \rightarrow[0,1)$ is monotonically decreasing function, then $T$ has a unique fixed point.

We now generalize both Theorem 1 and Theorem 3 .

THEOREM 4. Let $\alpha, \beta, \gamma$ be monotonically decreasing functions from $(0, \infty)$ into $[0,1)$ with $\alpha(t)+2 \beta(t)+2 \gamma(t)<1, t \in(0, \infty)$. Let $T$ be a selfmapping of $X$ such that the diagram of $T$ is closed and that

$$
\begin{aligned}
d(T x, T y) \leqslant & \alpha(d(x, y)) d(x, y)+ \\
& +\beta(d(x, y))[d(x, T x)+d(y, T y)]+ \\
& +\gamma(d(x, y))[d(x, T y)+d(y, T x)]
\end{aligned}
$$

for each $x \neq y \in X$, then $T$ has a unique fixed point.

Proof. Let $x_{0} \in X$ be arbitrary and let us consider $\left\{T^{n} x_{0}\right\}$. Suppose $T^{n-1} x_{0}$ $\neq T^{n} x_{0}$. Then for $n>1$ we have

$$
\begin{aligned}
d\left(T^{n} x_{0}, T^{n+1} x_{0}\right) \leqslant & \alpha\left(d\left(T^{n-1} x_{0}, T^{n} x_{0}\right)\right) d\left(T^{n-1} x_{0}, T^{n} x_{0}\right)+ \\
& +\beta\left(d\left(T^{n-1} x_{0}, T^{n} x_{0}\right)\right)\left[d\left(T^{n-1} x_{0}, T^{n} x_{0}\right)+d\left(T^{n} x_{0}, T^{n+1} x_{0}\right)\right]+ \\
& +\gamma\left(d\left(T^{n-1} x_{0}, T^{n} x_{0}\right)\right)\left[d\left(T^{n-1} x_{0}, T^{n+1} x_{0}\right)+d\left(T^{n} x_{0}, T^{n} x_{0}\right)\right.
\end{aligned}
$$

or

$$
d\left(T^{n} x_{0}, T^{n+1} x_{0}\right) \leqslant\left(\frac{\alpha+\beta+\gamma}{1-\beta-\gamma}\right) d\left(T^{n-1} x_{0}, T^{n} x_{0}\right)<d\left(T^{n-1} x_{0}, T^{n} x_{0}\right) .
$$

Hence $\left\{d\left(T^{n} x_{0}, T^{n+1} x_{0}\right)\right\}$ decreases. Let

$$
\lim _{n \rightarrow \infty} d\left(T^{n} x_{0}, T^{n+1} x_{0}\right)=S
$$

and suppose $S>0$. Take

$$
\frac{\alpha(S)+\beta(S)+\gamma(S)}{1-\beta(S)-\gamma(S)}=a .
$$

Then $d\left(T^{n} x_{0}, T^{m+1} x_{0}\right) \geqslant S$ implies

$$
\begin{aligned}
\frac{\alpha\left(d\left(T^{n} x_{0}, T^{n+1} x_{0}\right)\right)+\beta\left(d\left(T^{n} x_{0}, T^{n+1} x_{0}\right)\right)+\gamma\left(d\left(T^{n} x_{0}, T^{n+1} x_{0}\right)\right)}{1-\beta\left(d\left(T^{n} x_{0}, T^{n+1} x_{0}\right)\right)-\gamma\left(d\left(T^{n} x_{0}, T^{n+1} x_{0}\right)\right)} \\
\leqslant \frac{\alpha(S)+\beta(S)+\gamma(S)}{1-\beta(S)-\gamma(S)}=a \forall n .
\end{aligned}
$$

Hence $d\left(T^{n} x_{0}, T^{n+1} x_{0}\right) \leqslant a d\left(T^{n-1} x_{0}, T^{n} x_{0}\right) \leqslant \ldots \leqslant a^{n} d\left(x_{0}, T x_{0}\right)$ and $a^{n} d\left(x_{0}, T x_{0}\right) \rightarrow 0$ as $n \rightarrow \infty$, since $a<1$. Now we intend to show that $\left\{T^{n} x_{0}\right\}$ is Cauchy. Suppose $T^{n-1} x_{0} \neq T^{m-1} x_{0}$, then

$$
\begin{aligned}
d\left(T^{n} x_{0}, T^{m} x_{0}\right) \leqslant & \alpha\left(d\left(T^{n-1} x_{0}, T^{m-1} x_{0}\right)\right) d\left(T^{n-1} x_{0}, T^{m-1} x_{0}\right)+ \\
& +\beta\left(d\left(T^{n-1} x_{0}, T^{m-1} x_{0}\right)\right)\left[d\left(T^{n-1} x_{0}, T^{n} x_{0}\right)+d\left(7^{m-1} x_{0}, T^{m} x_{0}\right)\right]+ \\
& +\gamma\left(d\left(T^{n-1} x_{0}, T^{m-1} x_{0}\right)\right)\left[d\left(T^{n-1} x_{0}, T^{m} x_{0}\right)+d\left(T^{m-1} x_{0}, T^{n} \dot{x}_{0}\right)\right]
\end{aligned}
$$

i.e.,

$$
d\left(T^{n} x_{0}, T^{m} x_{0}\right) \leqslant\left(\frac{\alpha+\beta+\gamma}{1-\alpha-2 \gamma}\right) d\left(T^{n-1} x_{0}, T^{n} x_{0}\right)+\left(\frac{\alpha+\beta+\gamma}{1-\alpha-2 \gamma}\right) d\left(T^{m-1} x_{0}, T^{m} x_{0}\right)
$$

Let $\varepsilon>0$ be given. If $\alpha(\varepsilon)+\beta(\varepsilon)+\gamma(\varepsilon) \neq 0$, then we can find an $N$ such that

$$
d\left(T^{n-1} x_{0}, T^{n} x_{0}\right)<\frac{1}{2} \min \left\{\frac{(1-\alpha(\varepsilon)-2 \gamma(\varepsilon)) \varepsilon}{\alpha(\varepsilon)+\beta(\varepsilon)+\gamma(\varepsilon)}, 2 \varepsilon\right\}
$$

and

$$
d\left(T^{m-1} x_{0}, T^{m} x_{0}\right)<\frac{1}{2} \min \left\{\frac{(1-\alpha(\varepsilon)-2 \gamma(\varepsilon)) \varepsilon}{\alpha(\varepsilon)+\beta(\varepsilon)+\gamma(\varepsilon)}, 2 \varepsilon\right\}
$$

for all $n, m \geqslant N$. If $\alpha(\varepsilon)+\beta(\varepsilon)+\gamma(\varepsilon)=0$ for example, we require that for all $n, m \geqslant N$,

$$
d\left(T^{n-1} x_{0}, T^{n} x_{0}\right)<\varepsilon \text { and } d\left(T^{m-1} x_{0}, T^{m} x_{0}\right)<\varepsilon .
$$


Let us take any $n, m \geqslant N$. We wish to show that $d\left(T^{n} x_{0}, T^{m} x_{0}\right)<\varepsilon$. Assume $T^{n-1} x_{0}$ $\neq T^{m-1} x_{0}$. If $d\left(T^{n-1} x_{0}, T^{m-1} x_{0}\right) \geqslant \varepsilon$ then since $\alpha, \beta, \gamma$ are monotonically decreasing functions we have

$$
\begin{aligned}
& d\left(T^{n} x_{0}, T^{m} x_{0}\right) \\
& \leqslant \frac{\alpha(\varepsilon)+\beta(\varepsilon)+\gamma(\varepsilon)}{1-\alpha(\varepsilon)-2 \gamma(\varepsilon)} d\left(T^{n-1} x_{0}, T^{n} x_{0}\right)+\frac{\alpha(\varepsilon)+\beta(\varepsilon)+\gamma(\varepsilon)}{1-\alpha(\varepsilon)-2 \gamma(\varepsilon)} d\left(T^{m-1} x_{0}, T^{m} x_{0}\right)<\varepsilon . \\
& \text { On the otherhand } d\left(T^{n-1} x_{0}, T^{m-1} x_{0}\right)<\varepsilon \text { implies } \\
& d\left(T^{n} x_{0}, T^{m} x_{0}\right) \leqslant \alpha d\left(T^{n-1} x_{0}, T^{m-1} x_{0}\right)+\beta\left[d\left(T^{m-1} x_{0}, T^{m} x_{0}\right)+d\left(T^{n-1} x_{0}, T^{n} x_{0}\right)\right]+ \\
& +\gamma\left[d\left(T^{n-1} x_{0}, T^{m} x_{0}\right)+d\left(T^{m-1} x_{0}, T^{n} x_{0}\right)\right] \\
& <(\alpha+2 \beta+2 \gamma) \varepsilon<\varepsilon \text {. }
\end{aligned}
$$

Thus $\left\{T^{n} x_{0}\right\}$ is Cauchy. Since $X$ is a complete metric space, $\left\{T^{n} x_{0}\right\}$ converges to a point $\xi$ in $X$.

Now since the diagram. of $T$ is closed, we have $\lim T^{n+1} x_{0}=T \xi$. Thus $\xi$ is a fixed point of $T$.

We need prove now that $\xi$ is a unique fixed point of $T$. If possible let $\xi, \eta$, $\xi \neq \eta$ be two fixed point of $T$. Then

$$
d(\xi, \eta)=d(T \xi, T \eta) \leqslant[\alpha(d(\xi, \eta))+2 \gamma(d(\xi, \eta))] d(\xi, \eta),
$$

which gives a contradiction. Hence $\xi$ is a unique fixed point of $T$. This completes the proof.

We apply Theorem 4 to the following proposition which is a generalization of a result due to Nadler [9].

THeOREM 5. Let $T_{n}: X \rightarrow X$ be a function with at least one fixed point $a_{n}$ for each $n=1,2, \ldots$ and let $T_{0}: X \rightarrow X$ satisfy the hypotheses of Theorem 4 with the same $\alpha, \beta, \gamma$. If the sequence $T_{n}$ converges uniformly to $T_{0}$ then the sequence $a_{n}$ converges to $a_{0}$, the unique fixed point of $T_{0}$.

Proof. Assume $a_{n} \neq a_{0}$, then

$$
\begin{aligned}
d\left(a_{n}, a_{0}\right) \leqslant & d\left(T_{n} a_{n}, T_{0} a_{n}\right)+d\left(T_{0} a_{n}, T_{0} a_{0}\right) \\
\leqslant & d\left(T_{n} a_{n}, T_{0} a_{n}\right)+\alpha\left(d\left(a_{n}, a_{0}\right)\right) d\left(a_{n}, a_{0}\right)+ \\
& +\beta\left(d\left(a_{n}, a_{0}\right)\right)\left[d\left(a_{n}, T_{0} a_{n}\right)+d\left(a_{0}, T_{0} a_{0}\right)\right]+ \\
& +\gamma\left(d\left(a_{n}, a_{0}\right)\right)\left[d\left(a_{n}, T_{0} a_{0}\right)+d\left(a_{0}, T_{0} a_{n}\right)\right] .
\end{aligned}
$$

Hence we get

$$
d\left(a_{n}, a_{0}\right) \leqslant\left[\frac{1+\beta\left(d\left(a_{n}, a_{0}\right)\right)+\gamma\left(d\left(a_{n}, a_{0}\right)\right)}{1-\alpha\left(d\left(a_{n}, a_{0}\right)\right)-2 \gamma\left(d\left(a_{n}, a_{0}\right)\right)}\right] d\left(T_{n} a_{n}, T_{0} a_{n}\right) .
$$

Let $\varepsilon_{0}>0$ be arbitrary and choose $\varepsilon_{1}>0$ such that

$$
\varepsilon_{1}<\left[\frac{1-\alpha\left(\varepsilon_{0}\right)-2 \gamma\left(\varepsilon_{0}^{*}\right)}{1+\beta\left(\varepsilon_{0}\right)+\gamma\left(\varepsilon_{0}\right)}\right] \varepsilon_{0} .
$$

Since $\left\{T_{n}\right\}_{n=1}^{\infty}$ converges uniformly to $T_{0}$, so there is a $K$ and a positive integer $N$ such that for all $K \geqslant N$ and for all $x, d\left(T_{K} x, T_{0} x\right)<\varepsilon_{1}$.

CLAIM. For all $i \geqslant N, d\left(a_{i}, a_{0}\right)<\varepsilon_{0}$

Suppose not. Then there exists a $j \geqslant N$ such that

$$
d\left(a_{j}, a_{0}\right) \geqslant \varepsilon_{0} .
$$

Since $\alpha, \beta, \gamma$ are monotonically decreasing functions, so by (11), the relation (10) gives

$$
d\left(a_{j}, a_{0}\right) \leqslant \frac{1+\beta\left(\varepsilon_{0}\right)+\gamma\left(\varepsilon_{0}\right)}{1-\alpha\left(\varepsilon_{0}\right)-2 \gamma\left(\varepsilon_{0}\right)} d\left(T_{j} a_{j}, T_{0} a_{j}\right)<\varepsilon_{0}
$$

which contradicts (10). Therefore $a_{n} \rightarrow a_{0}$.

THEOREM 6 (cf. [6]). Let $\left(X, d_{n}\right)$ be a complete metric space for each $n=0,1,2,$. and suppose $\left\{d_{n}\right\}_{n=1}^{\infty}$ converges uniformly to $d_{0}$. Let $T_{n}:\left(X, d_{n}\right) \rightarrow\left(X, d_{n}\right)$ satisfy the hypotheses of Theorem 4 with the same continuous $\alpha, \beta, \gamma$ for all $n=1,2, \ldots$ and let $a_{n}$ be the fixed points of $T_{n}$ for $n=1,2, \ldots$ If a mapping $T_{0}:\left(X, d_{0}\right) \rightarrow\left(X, d_{0}\right)$ is defined as the $d_{0}$ - pointwise limits of $T_{n}$, then $a_{n} \rightarrow a_{0}$, the unique fixed point of $T_{0}$.

Proof. First we shall show that $T_{0}$ satisfies (9) with respect to $d_{0}$. Now

$$
\begin{aligned}
d_{0}\left(T_{0} x, T_{0} y\right) & \leqslant d_{0}\left(T_{0} x, T_{n} x\right)+d_{0}\left(T_{n} x, T_{n} y\right)+d_{0}\left(T_{n} y, T_{0} y\right) \\
& \leqslant d_{0}\left(T_{0} x, T_{n} x\right)+d_{n}\left(T_{n} x, T_{n} y\right)+\varepsilon+d_{0}\left(T_{n} y, T_{0} y\right)
\end{aligned}
$$

(the latter inequality is valid for $n \geqslant N$ )

$$
\begin{aligned}
\leqslant & d_{0}\left(T_{0} x, T_{n} x\right)+\alpha\left(d_{n}(x, y)\right) d_{n}(x, y)+ \\
& +\beta\left(d_{n}(x, y)\right)\left[d_{n}\left(x, T_{n} x\right)+d_{n}\left(y, T_{n} y\right)\right]+ \\
& +v\left(d_{n}(x, y)\right)\left[d_{n}\left(x, T_{n} y\right)+d_{n}\left(y, T_{n} x\right)\right]+ \\
& +d_{0}\left(T_{n} y, T_{0} y\right)+\varepsilon \\
\leqslant & d_{0}\left(T_{0} x, T_{n} x\right)+\alpha\left(d_{n}(x, y)\right)\left[d_{0}(x, y)+\varepsilon\right]+ \\
& +\beta\left(d_{n}(x, y)\right)\left[d_{0}\left(x, T_{n} x\right)+\varepsilon+d_{0}\left(y, T_{n} y\right)+\varepsilon\right]+ \\
& +\gamma\left(d_{n}(x, y)\right)\left[d_{0}\left(x, T_{n} y\right)+\varepsilon+d_{0}\left(y, T_{n} x\right)+\varepsilon\right]+ \\
& +d_{0}\left(T_{n} y, T_{0} y\right)+\varepsilon .
\end{aligned}
$$

As $n \rightarrow \infty$ we get

$$
\begin{aligned}
d_{0}\left(T_{0} x, T_{0} y\right) \leqslant & \alpha\left(d_{0}(x, y)\right)\left[d_{0}(x, y)+\varepsilon\right] \\
& +\beta\left(d_{0}(x, y)\right)\left[d_{0}\left(x, T_{0} x\right)+\varepsilon+d_{0}\left(y, T_{0} y\right)+\varepsilon\right] \\
& +\gamma\left(d_{0}(x, y)\right)\left[d_{0}\left(x, T_{0} y\right)+\varepsilon+d_{0}\left(y, T_{0} x\right)+\varepsilon\right]+\varepsilon .
\end{aligned}
$$


Since this is true for every $\varepsilon>0$ we get

$$
d_{0}\left(T_{0} x, T_{0} y\right) \leqslant \alpha\left(d_{0}(x, y)\right) d_{0}(x, y)+\beta\left(d_{0}(x, y)\right)\left[d_{0}\left(x, T_{0} x\right)+d_{0}\left(y, T_{0} y\right)\right]+
$$

$$
+\gamma\left(d_{0}(x, y)\right)\left[d_{0}\left(x, T_{0} y\right)+d_{0}\left(y, T_{0} x\right)\right] .
$$

Now

$$
\begin{aligned}
d_{0}\left(a_{n}, a_{0}\right) \leqslant & d_{0}\left(T_{n} a_{n}, T_{n} a_{0}\right)+d_{0}\left(T_{n} a_{0}, T_{0} a_{0}\right) \\
\leqslant & d_{n}\left(T_{n} a_{n}, T_{n} a_{0}\right)+\varepsilon+d_{0}\left(T_{n} a_{0}, T_{0} a_{0}\right) \\
\leqslant & \alpha\left(d_{n}\left(a_{n}, a_{0}\right)\right) d_{n}\left(a_{n}, a_{0}\right)+\beta\left(d_{n}\left(a_{n}, a_{0}\right)\right)\left[d_{n}\left(a_{n}, T_{n} a_{n}\right)+d_{n}\left(a_{0}, T_{n} a_{0}\right)\right]+ \\
& +\gamma\left(d_{n}\left(a_{n}, a_{0}\right)\right)\left[d_{n}\left(a_{n}, T_{n} a_{0}\right)+d_{n}\left(a_{0}, T_{n} a_{n}\right)\right]+\varepsilon+d_{0}\left(T_{n} a_{0}, T_{0} a_{0}\right)
\end{aligned}
$$

$\leqslant \alpha\left(d_{n}\left(a_{n}, a_{0}\right)\right)\left[d_{0}\left(a_{n}, a_{0}\right)+\varepsilon\right]+\beta\left(d_{n}\left(a_{n}, a_{0}\right)\right)\left[d_{0}\left(T_{n} a_{0}, T_{0} a_{0}\right)+\varepsilon\right]+$

$+\gamma\left(d_{n}\left(a_{n}, a_{0}\right)\right)\left[d_{0}\left(a_{n}, a_{0}\right)+2 \varepsilon+d_{0}\left(T_{0} a_{0}, T_{n} a_{0}\right)+\varepsilon\right]$

$+\varepsilon+d_{0}\left(T_{n} a_{0}, T_{0} a_{0}\right)$.

Hence

$$
\begin{aligned}
d_{0}\left(a_{n}, a_{0}\right) \leqslant & \alpha \varepsilon+\beta\left(d_{n}\left(a_{n}, a_{0}\right)\right)\left[d_{0}\left(T_{n} a_{0}, T_{0} a_{0}\right)+\varepsilon\right]+ \\
& +\gamma\left(d_{n}\left(a_{n}, a_{0}\right)\right)\left[d_{0}\left(T_{n} a_{0}, T_{0} a_{0}\right)+3 \varepsilon\right]+ \\
& +\frac{\varepsilon+d_{0}\left(T_{n} a_{0}, T_{0} a_{0}\right)}{1-\alpha\left(d_{n}\left(a_{n}, a_{0}\right)\right)-2 \gamma\left(d_{n}\left(a_{n}, a_{0}\right)\right)}
\end{aligned}
$$

Thus for any $\varepsilon>0$ there exists an $N(\varepsilon)$ such that $n \geqslant N(\varepsilon)$ implies that

$$
d_{0}\left(a_{n}, a_{0}\right) \leqslant \frac{3 d_{0}\left(T_{n} a_{0}, T_{0} a_{0}\right)+6 \varepsilon}{1-\alpha\left(d_{0}\right.} \frac{\left.\left(a_{n}, a_{0}\right)\right)-2 \gamma\left(d_{0}\left(a_{n}, a_{0}\right)\right)}{.} .
$$

Let $\varepsilon_{1}>0$ be given. Take $\varepsilon=\frac{1}{12}\left[1-\alpha\left(\varepsilon_{1}\right)-2 \gamma\left(\varepsilon_{1}\right)\right] \varepsilon_{1}$. Let $n$ be so large so that $n \geqslant N(\varepsilon)$ and $d_{0}\left(T_{n} a_{0}, T_{0} a_{0}\right)<\frac{1}{6}\left[1-\alpha\left(\varepsilon_{1}\right)-2 \gamma\left(\varepsilon_{1}\right)\right] \varepsilon_{1}$. For these $n$, if $d_{0}\left(a_{n}, a_{0}\right) \geqslant \varepsilon_{1}$, we get $d_{0}\left(a_{n}, a_{0}\right)<\varepsilon_{1}$ and we arrive at a contradiction. This completes the proof.

2. There exists a local form of Banach's fixed point theorem [7]. Its analogue is THEOREM 7 (Localization of Theorem 2). Let

$$
S\left(x_{0}, r\right)=\left\{x \in X: d\left(x, x_{0}\right) \leqslant r\right\}
$$

be a sphere in $X$ and let $T: X \rightarrow X$ be such that for every $x, y \in S\left(x_{0}, r\right)$ we have

$$
d(T x, T y) \leqslant \alpha d(x, y)+\beta d(x, T x)+\gamma d(y, T y)+\delta[d(x, T y)+d(y, T x)]
$$

for some $\alpha, \beta, \gamma, \delta \in R_{+}$with $\alpha+\beta+\gamma+2 \delta<1$. If

(12) $\quad d\left(x_{0}, T x_{0}\right) \leqslant(1-\lambda) r$ where $\lambda=(\alpha+\beta+\delta) /(1-\gamma-\delta)$,

then $T$ has a unique fixed point.
Proof. By (12) $x_{1}=T x_{0} \in S\left(x_{0}, r\right)$. Now

$$
d\left(x_{1}, x_{2}\right)=d\left(T x_{0}, T x_{1}\right)
$$

$\leqslant \alpha d\left(x_{0}, x_{1}\right)+\beta d\left(x_{0}, T x_{0}\right)+\gamma d\left(x_{1}, T x_{1}\right)+\delta\left[d\left(x_{0}, T x_{1}\right)+d\left(x_{1}, T x_{0}\right)\right]$,

i.e., $d\left(x_{1}, x_{2}\right) \leqslant(1-\lambda) \lambda r$. Hence $d\left(x_{0}, x_{2}\right) \leqslant(1+\lambda)(1-\lambda) r$. Suppose

$$
d\left(x_{0}, x_{n}\right) \leqslant\left(1+\lambda+\ldots+\lambda^{n-1}\right)(1-\lambda) r
$$

and that

$$
d\left(x_{n-1}, x_{n}\right) \leqslant \lambda^{n-1}(1-\lambda) r, \quad x_{n}=T x_{n-1}, n=1,2, \ldots
$$

Then

$$
\begin{aligned}
d\left(x_{n}, T x_{n}\right)= & d\left(T x_{n-1}, T x_{n}\right) \\
\leqslant & \alpha d\left(x_{n-1}, x_{n}\right)+\beta d\left(x_{n-1}, T x_{n-1}\right)+\gamma d\left(x_{n}, T x_{n}\right) \\
& +\delta\left[d\left(x_{n-1}, T x_{n}\right)+d\left(x_{n}, T x_{n-1}\right)\right],
\end{aligned}
$$

i.e., $d\left(x_{n}, x_{n+1}\right) \leqslant(1-\lambda) \lambda^{n} r$. This implies

$$
d\left(x_{0}, x_{n+1}\right) \leqslant\left(1+\lambda+\ldots+\lambda^{n}\right)(1-\lambda) r \leqslant r .
$$

Thus the sequence $x_{0}, x_{n+1}=T x_{n}, n \geqslant 0$, is contained in $S$. Again

$$
d\left(x_{n}, x_{m}\right) \leqslant\left(\lambda^{n}+\lambda^{n+1}+\ldots+\lambda^{m-1}\right)(1-\lambda) r \leqslant \lambda^{n} r \rightarrow 0 \text { as } n \rightarrow \infty .
$$

Since $S$ is also complete, $\lim x_{n}=\xi$ for some $\xi \in S$. But

$$
\begin{aligned}
d\left(x_{n+1}, T \xi\right)= & d\left(T x_{n}, T \xi\right) \\
\leqslant & \alpha d\left(x_{n}, \xi\right)+\beta d\left(x_{n}, T x_{n}\right)+\gamma d(\xi, T \xi)+\delta\left[d\left(x_{n}, T \xi\right)+d\left(\xi, T x_{n}\right)\right] \\
\leqslant & \alpha d\left(x_{n}, \xi\right)+\beta d\left(x_{n}, x_{n+1}\right)+\gamma d\left(x_{n+1}, T \xi\right)+\delta d\left(x_{n}, x_{n+1}\right) \\
& +\delta d\left(x_{n+1}, T \xi\right)+\delta d\left(\xi, x_{n}\right)+\delta d\left(x_{n+1}, x_{n}\right)+\gamma d\left(\xi, x_{n+1}\right) .
\end{aligned}
$$

Hence $x_{n+1} \rightarrow T \xi$ as $n \rightarrow \infty$, i.e., $\xi$ is a fixed point of $T$. Uniqueness of $\xi$ is obvious.

3. Ciric's fixed point theorem can be extended to multivalued mappings. Let $\mathscr{F}(X)$ denote the family of all nonempty closed and bounded subsets of a given metric space $X, H(A, B)$ the Hausdorff metric [8] for $A, B \in \mathscr{F}(X)$ and let $D(x, A)$ $=\inf \{d(x, y): y \in A$, where $A \in \mathscr{F}(X)\}$.

THEOREM 8. Let $F: X \rightarrow X$ be a multivalued function such that the diagram of $F$ is closed and that

$$
\begin{aligned}
H(F(x), F(y)) \leqslant & \alpha d(x, y)+\beta[D(x, F(x))+D(y, F(y))]+ \\
& +\gamma[D(x, F(y))+D(y, F(x))]
\end{aligned}
$$

where $\alpha>0, \beta>0, \gamma>0$ with $\alpha+2 \beta+2 \gamma<1$. Then $F$ has a fixed point. 
Proof. Pick any $x_{0} \in X$ and choose $x_{1} \in F\left(x_{0}\right)$. If $H\left(F\left(x_{0}\right), F\left(x_{1}\right)\right)=0$ then $F\left(x_{0}\right)=F\left(x_{1}\right)$ and hence $x_{1} \in F\left(x_{1}\right)$, i.e., $x_{1}$ is a fixed point of $F$. Therefore we may assume that $H\left(F\left(x_{0}\right), F\left(x_{1}\right)\right)>0$. By definition, if $h>H\left(F\left(x_{0}\right), F\left(x_{1}\right)\right)$, there exists $x_{2} \in F\left(x_{1}\right)$ such that $d\left(x_{1}, x_{2}\right)<h$. Let $h=\lambda_{1}^{-1} H\left(F\left(x_{0}\right), F\left(x_{1}\right)\right)$ where $\lambda_{1}$ $=(\alpha+2 \beta+2 \gamma)^{1 / 2}\left(\lambda_{1}<1\right.$ and that we may assume $\left.\lambda_{1}>0\right)$. Then

$$
\begin{aligned}
d\left(x_{1}, x_{2}\right) \leqslant & \lambda_{1}^{-1} H\left(F\left(x_{0}\right), F\left(x_{1}\right)\right) \\
\leqslant & \lambda_{1}^{-1}\left[\alpha d\left(x_{0}, x_{1}\right)+\beta\left[D\left(x_{0}, F\left(x_{0}\right)\right)+D\left(x_{1}, F\left(x_{1}\right)\right)\right]\right. \\
& \left.+\gamma\left[D\left(x_{0}, F\left(x_{1}\right)\right)+D\left(x_{1}, F\left(x_{0}\right)\right)\right]\right] \\
\leqslant & \lambda_{1}^{-1}\left[\alpha d\left(x_{0}, x_{1}\right)+\beta\left[d\left(x_{0}, x_{1}\right)+d\left(x_{1}, x_{2}\right)\right]+\gamma\left[d\left(x_{0}, x_{2}\right)\right]\right],
\end{aligned}
$$

i.e.,

$$
d\left(x_{1}, x_{2}\right) \leqslant q d\left(x_{0}, x_{1}\right) \quad \text { where } \quad q=\lambda_{1}^{-1}(\alpha+\beta+\gamma) /\left(1-\lambda_{1}^{-1} \beta-\lambda_{1}^{-1} \gamma\right)<1 .
$$
Let us suppose that $\left(F\left(x_{i-1}\right), F\left(x_{i}\right)\right)>0$ for $i \geqslant 2$. By induction we get $x_{i+1}$
$\in F\left(x_{i}\right)$ such that

$$
d\left(x_{i}, x_{i+1}\right) \leqslant q d\left(x_{i-1}, x_{i}\right) \leqslant \ldots \leqslant q^{i} d\left(x_{0}, x_{1}\right) .
$$

Now, if $n>m$

$$
\begin{gathered}
d\left(x_{n}, x_{m}\right) \leqslant\left(q^{m}+q^{m+1}+\ldots+q^{n-1}\right) d\left(x_{0}, x_{1}\right) \\
\leqslant \frac{q^{m}}{1-q} d\left(x_{0}, x_{1}\right) \rightarrow 0 \quad \text { as } m \rightarrow \infty .
\end{gathered}
$$

Thus the sequence $\left\{x_{i}\right\}_{i=1}^{\infty}$ is a Cauchy sequence and since $X$ is complete, $\left\{x_{i}\right\}_{i=1}^{\infty}$ converges to $P_{0} \in X$. Since the diagram of $F$ is closed, $\lim \left\{F\left(x_{i}\right)\right\}_{i=1}^{\infty}=F\left(P_{0}\right)$ But $x_{i} \in F\left(x_{i-1}\right)$ for all $i=1,2, \ldots$ Hence $P_{0} \in F\left(P_{0}\right)$. This completes the proof.

\section{References}

[1] L. B. Ciric, Generalized contractions and fixed point theorems, Publ. Inst. Math. 12 (1971), pp. $20-26$.

[2] S. Banach, Sur les opérations dans ensembles abstraits et leur applications aux équations intégrales, Fund. Math. 3 (1922), pp. 133-181.

[3] R. Kannan, Some results on fixed points, Bull. Cal. Math. Soc. 60 (1968), pp. 71-76. pp. $727-730$.

[5] E. Rakotch, A note on contacive ma

[6] E. Rakotch, A note on contractive mappings, Proc. Amer. Math. Soc. 13 (1962), pp. 459-465. R. B. Fraser, Jr. and S. B. Nadler, Jr., Sequences of contractive maps and fixed points,
Pacific J. Math. 51 (1969), pp. 659-667. 7] L.A. Lusternik and V.J.
[8] S. B. Nadler Jr., Multivalued contraction mappings, Pacific J. Math. 30 (1969), pp. 475-488. [9] - Sequences of contractions and fixed points, Pacific J. Math 27 (1968), pp. 579-585.

[10] S. Reich, Kannan's fixed point theorem, Boll. U. M. I 4 (1971), pp. 1-11.

[11] B. K. Ray, On common fixed points and sequence of mappings, Rev. Roum. Math. Pures et Appliquées (to appear).

\section{DEPARTMENT OF MATHEMATICS} REGIONAL ENGINERING COLLEGE Durgapur, India

Accepté par la Rédaction le 3. 2. 1975 